\title{
BMJ Open Self-directed multimedia process for delivering participant informed consent
}

To cite: Chapman N, McWhirter R, Armstrong MK, et al. Self-directed multimedia process for delivering participant informed consent. BMJ Open 2020;10:e036977. doi:10.1136/ bmjopen-2020-036977

- Prepublication history and additional material for this paper are available online. To view these files, please visit the journal online (http://dx.doi org/10.1136/bmjopen-2020036977).

Received 15 January 2020 Revised 14 April 2020 Accepted 08 June 2020

Check for updates

(c) Author(s) (or their employer(s)) 2020. Re-use permitted under CC BY-NC. No commercial re-use. See rights and permissions. Published by BMJ.

${ }^{1}$ Menzies Institute for Medical Research, College of Health and Medicine, University of Tasmania, Hobart, Tasmania, Australia

${ }^{2}$ Faculty of Law, Centre for Law and Genetics, University of Tasmania, Hobart, Tasmania, Australia

Correspondence to

Professor James E Sharman; James.Sharman@utas.edu.au

\author{
Niamh Chapman (D) , ${ }^{1}$ Rebekah McWhirter, ${ }^{1,2}$ Matthew K Armstrong, ${ }^{1}$ \\ Ricardo Fonseca, ${ }^{1}$ Julie A Campbell (D) , ${ }^{1}$ Mark Nelson, ${ }^{1}$ Martin G Schultz, ${ }^{1}$ \\ James E Sharman ${ }^{1}$
}

\section{ABSTRACT}

Objective Obtaining informed consent is a cornerstone requirement of conducting ethical research. Traditional paper-based consent is often excessively lengthy and may fail to achieve the desired participant understanding of study requirements. Multimedia tools including video and audio may be a useful alternative. This study aimed to determine the efficacy, usability and acceptability of selfdirected multimedia delivery of participant consent. Design It is a single-centre, randomised, prospective study to determine the efficacy, usability and acceptability of a self-directed multimedia consent process (intervention) compared with the traditional paper-based approach (control). The intervention was free of research staff, with computer-based finger-signed consent.

Setting Pathology blood collection services in Tasmania, Australia.

Participants 298 participants ( $63 \pm 8$ years; $51 \%$ female individuals) referred from general practice were randomised to intervention $(n=146)$ and control $(n=152)$. Outcome measures Efficacy, usability and acceptability of the allocated consent process were assessed by a questionnaire.

Results All participants successfully completed the allocated interventions. Efficacy parameters were higher among intervention participants, including a better understanding of study requirements compared with controls ( $p<0.05$ all). Intervention participants were more likely to engage with the study information and spend more time on the consent process $(p=<0.001$ and $p=0.006$, respectively). Both groups reported similar levels of acceptability, although more control participants reported that the study information was too long ( $24 \%$ vs $14 \% ; p=0.020$ ).

Conclusion A self-directed multimedia consent process is effective for achieving participant understanding and obtaining consent free of research staff. Thus, multimedia represents a viable method to reduce the burden on researchers, meet participant needs and achieve informed consent in clinical research.

\section{INTRODUCTION}

Informed consent is a cornerstone procedure of ethically conducted medical research. Consent processes aim to ensure that potential participants are fully informed prior to deciding to take part in the research. Guidelines emphasise the need for full disclosure

\section{Strengths and limitations of this study}

To our knowledge, this is the largest randomised evaluation of a self-directed multimedia consent process.

- Multimedia consent tools were developed in collaboration with community members.

- Self-directed multimedia was an acceptable, efficient and effective alternative to traditional consent processes in medical research.

- Generalisability of the findings will need to be confirmed in further studies.

of study information including the aims, requirements, risks, benefits, funding and conflicts of interest, with the view that more information facilitates better-informed decision making. ${ }^{1-3}$ However, this has resulted in lengthy consent processes that are burdensome for both researchers and participants while often failing to achieve the desired level of participant understanding. ${ }^{4-11}$ Indeed, as few as $50 \%$ of participants understand study information, including associated risks and that participation is voluntary. ${ }^{5}$ These shortcomings, as well as the emergence of complex contemporary methods, including biobanking, gene sequencing, linked data, remote research and large-scale trials often spanning multiple countries, have led to calls to update consent guidelines to more appropriately reflect the modern research landscape. $^{12-15}$

Self-directed multimedia delivery of information via video and audio platforms may offer an effective alternative or complementary tool to traditional consent processes. Previous reviews evaluating the efficacy of multimedia tools in the consent process have been inconclusive. ${ }^{41617}$ This ambiguity may be due to heterogeneous study designs and population characteristics. Moreover, previous research focused on using multimedia to augment traditional research consent processes, with a researcher present, 
rather than multimedia as a standalone and self-directed process, making it difficult to discern the generalisability and utility of a self-directed multimedia process for consent. In any case, there seems to be good acceptability and usability of multimedia tools used within the consent process with respect to participant satisfaction and facilitating recruitment, but also for understanding information in a non-research (clinical) setting. ${ }^{16-18}$

As far as we are aware, there has never been a study to determine if consent for participation in research can be appropriately delivered in the absence of research staff using a self-directed multimedia process compared with the traditional paper-based approach in the presence of research staff. This study sought to determine this during the consent process for people being recruited to participate in a clinical research project that focused on cardiovascular risk assessment.

\section{METHODS}

\section{Study protocol}

This research was undertaken in the context of a study testing the use of a computer-based application (app) to gather information for the assessment of absolute cardiovascular disease risk within a clinical setting. ${ }^{19}$ Participants referred by a general practitioner to pathology services were approached for involvement in the cardiovascular risk assessment study by the pathology services receptionist. Inclusion criteria for participation included those with a referral for a full lipid profile aged between 45 and 74 years in accordance with absolute cardiovascular risk assessment guidelines. ${ }^{20}$ Participants who were interested in involvement in the cardiovascular risk assessment study were randomised to receive self-directed multimedia consent (intervention) or traditional paperbased consent with a researcher (control) (figure 1). Due to the setting of the study, field notes were used to collect data on why participants did not take part after initial eligibility screening.

Both groups received a short demonstration on how to use the app. The demonstration was quick with rudimentary instructions provided as it was intended to be delivered by pathology staff in under a minute who would then resume normal clinical duties. The intervention group was shown how to play the study video and audio and advised to engage with the information until they had decided if they wanted to take part, at which point they could provide their consent or leave without taking part. The control group was provided with the paper-based information sheet by a researcher, advised to read and asked if they needed assistance or had any questions as per conventional consent processes. Both groups provided signed consent using their finger on a touchscreen monitor via the app to proceed to the cardiovascular assessment. Immediately after the app cardiovascular risk assessment, each participant was asked to complete a questionnaire to evaluate the efficacy, usability and acceptability of the consent process they had undertaken.

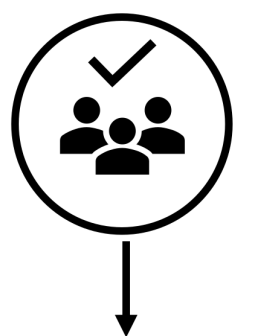

Referred patients screened for eligibility at reception. Eligible participants received study postcard ( $<150$ words).

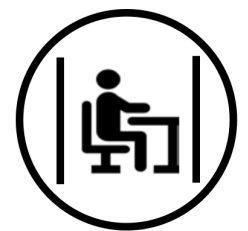

Participants directed to a private booth to participate after being randomly allocated to multimedia intervention or control consent processes.

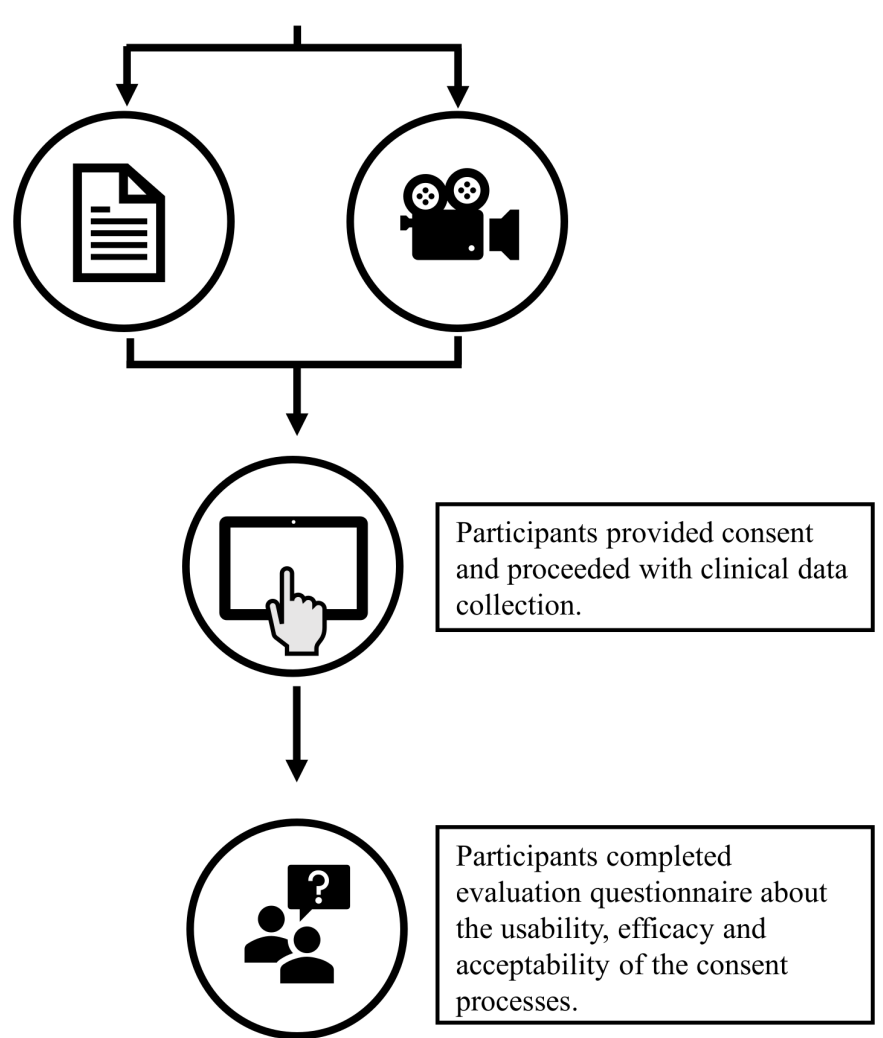

Figure 1 Flow diagram of study protocol.

\section{Randomisation}

Referred patients who met the criteria for participation received a postcard that contained basic information about the study and contact details for more information (online supplementary 1, Study postcard). A total of 831 participants were identified as eligible for participation in the cardiovascular risk assessment study; from these, 303 were randomised to participate (figure 2). Randomisation was determined by a computer program on a 1:1 ratio prior to recruitment. It was not possible to blind participants to their allocated interventions because multimedia was obviously different from paper-based consent.

Delivery of paper-based consent process as the study control Control participants received a two-page paper-based study participant information sheet compliant with 


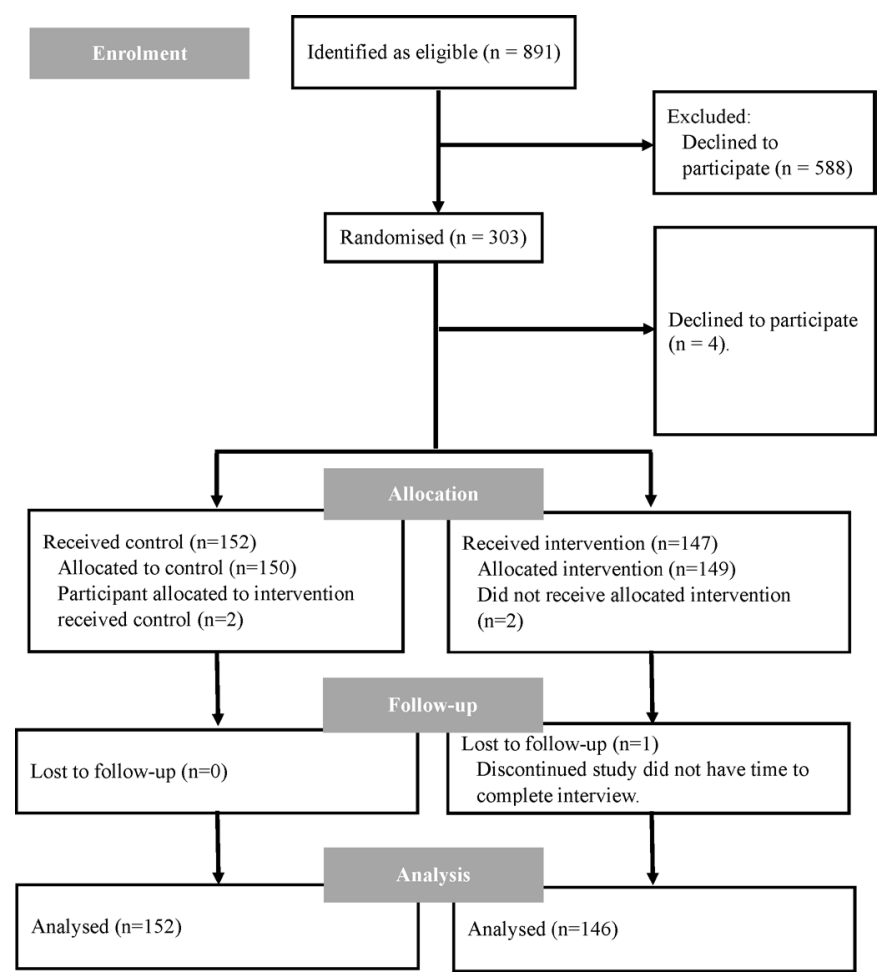

Figure 2 Participant flow diagram.

the requirements of the National Health and Medical Research Council and Australian Research Council, National Statement on Ethical Conduct in Human Research. ${ }^{1}$ The first page provided information on the aims, participation requirements and why participants were invited to take part. The second page detailed the risks, benefits, funding sources, ethical approval and privacy protections. The control consent process involved the participant being asked to read the information sheet in the presence of a researcher who provided further information and answered questions as requested (as per usual practice).

\section{Delivery of multimedia consent process as the study intervention}

Intervention participants received study participation information via multimedia approach using a $3 \mathrm{~min}$ animated video and separate audio content using the same terminology and content as the paper-based study participant information sheet. The study video was congruent with the first page of the information sheet and focused on the aims and requirements of the study (online supplementary 2, Study video). The separate audio content was congruent with the second page of the information sheet and provided information on study funding, ethical approval, risks and benefits associated with participation and privacy protection, which was clearly labelled. Each audio segment was approximately $30 \mathrm{~s}$ in duration. Participants were shown how to play the audio content as part of the app demonstration. A multidisciplinary team of research staff, graphic designers and communications staff developed the study video through an iterative approach including feedback from community members typical of the target demographic.

\section{Patient and public involvement}

Community members reviewed and contributed to all aspects of study materials including the questionnaires, multimedia and paper-based study information and advised on the content that was included in the final version. An iterative process was undertaken with community advisors to develop consent materials, with initial drafts completed by researchers. Community advisors provided several rounds of feedback (and final approval) on all consent materials, including the information sheet, postcard, video and audio recordings.

\section{Setting and consent environment}

All study procedures took place on the premises of pathology services. A purpose-built booth was designed for the study (figure 1). The study booth provided a private environment for the consent process and clinical data collection. The booth contained a bench with the computer that delivered the study app, a chair and a curtain for privacy.

\section{Assessment of consent process}

The evaluation questionnaire was delivered by a researcher at a separate workstation after participants completed all study processes in the booth. A 12-item questionnaire was used to assess efficacy, usability and acceptability of the consent process. The questionnaire was mixed methods with dichotomous and multiple-choice questions, each with a comment box for open-ended responses.

\section{Efficacy and usability of the consent process}

The effectiveness of the allocated consent processes to inform participants about the study was assessed via two measures: (1) the extent to which participants understood participation was voluntary and (2) participant understanding of specific aspects of study participation by true or false questions. Four measures denoting userfriendliness of the allocated consent processes were used to indicate usability: (1) participant engagement with the study information by reading, watching or listening; (2) participant perceived understanding of the study; (3) successful completion of the consent process and (4) the time taken to complete the consent process. The app automatically recorded the time for both groups as the app set-up and demonstration took place before the consent process. The time included the set-up, the consent process and the cardiovascular assessment questionnaire. All other parameters were measured by a selfreport questionnaire.

\section{Acceptability of the consent process}

Three indicators of acceptability of the consent process were used: (1) was there sufficient information available to give consent, (2) were participants satisfied with the length of the study information; and (3) what was the 
Table 1 Sociodemographic and clinical characteristics of study participants randomised to multimedia intervention or control delivery of study information and informed consent

\begin{tabular}{|c|c|c|}
\hline Variable & $\begin{array}{l}\text { Control } \\
(n=152)\end{array}$ & $\begin{array}{l}\text { Intervention } \\
(n=146)\end{array}$ \\
\hline Age (years) & $63 \pm 8$ & $63 \pm 7$ \\
\hline Male, n (\%) & $76(50)$ & $70(48)$ \\
\hline \multicolumn{3}{|l|}{ Education, n (\%) } \\
\hline High school & $37(24)$ & $31(21)$ \\
\hline $\begin{array}{l}\text { Certificate, diploma or } \\
\text { apprenticeship }\end{array}$ & 24 (16) & $31(21)$ \\
\hline $\begin{array}{l}\text { University degree or } \\
\text { higher }\end{array}$ & $81(53)$ & $76(52)$ \\
\hline \multicolumn{3}{|l|}{ Employment, n (\%) } \\
\hline Employed & $67(44)$ & $64(44)$ \\
\hline Retired & $56(37)$ & $58(37)$ \\
\hline Other & $18(12)$ & $13(9)$ \\
\hline \multicolumn{3}{|l|}{ Ethnicity, n (\%) } \\
\hline White & $131(86)$ & $131(90)$ \\
\hline $\begin{array}{l}\text { Aboriginal or Torres } \\
\text { Strait Islander }\end{array}$ & $1(1)$ & $2(1)$ \\
\hline Asian & $3(1)$ & $4(2)$ \\
\hline Other & $3(1)$ & $1(1)$ \\
\hline
\end{tabular}

Data are expressed as a percentage of the total sample size or mean \pm SD. Response rates varied from 135 to 152 for control and 133 to 146 for intervention.

No significant differences were observed between the groups.

preferred method of information delivery for deciding to take part in the research.

\section{Data analysis}

Data are presented as mean and SD or percentage of the total sample. For the comparison of categorical variables, percentage differences were tested using the $\chi^{2}$ test; the t-test was used for continuous variables. For all statistical tests, a $p$ value of $<0.05$ was considered significant. The analysis was conducted by a researcher blinded to allocation. Analyses were performed using Stata V.16.1 (StataCorp).

\section{RESULTS \\ Participant characteristics}

There were no differences in sociodemographic characteristics between the intervention and control groups (table 1). Participants were predominantly white and middle-older aged. Half of the participants had completed an undergraduate degree or higher, and a quarter was in full-time employment. From field notes, the main reason participants did not progress from eligibility screening to study participation was due to time constraints as many were attending pathology services before going to work.

\section{Efficacy and usability of multimedia intervention versus control}

Intervention participants demonstrated a better understanding of the follow-up requirements and data sharing practices of the study compared with control participants (table $2, \mathrm{p}<0.001$ and $\mathrm{p}=0.025$, respectively). Intervention participants were more likely to spend more time on the consent process and study questionnaire $(\mathrm{p}=0.006)$. Altogether, more intervention participants engaged with any form of study information compared with control participants. However, when the section of the information sheet that was congruent with the audio component was compared, only $9 \%$ of intervention participants listened to the separate audio and $35 \%$ of control participants read the second page of the information sheet.

Table 2 Efficacy and usability of the informed consent process of study participants randomised to multimedia intervention or control delivery of study information and informed consent

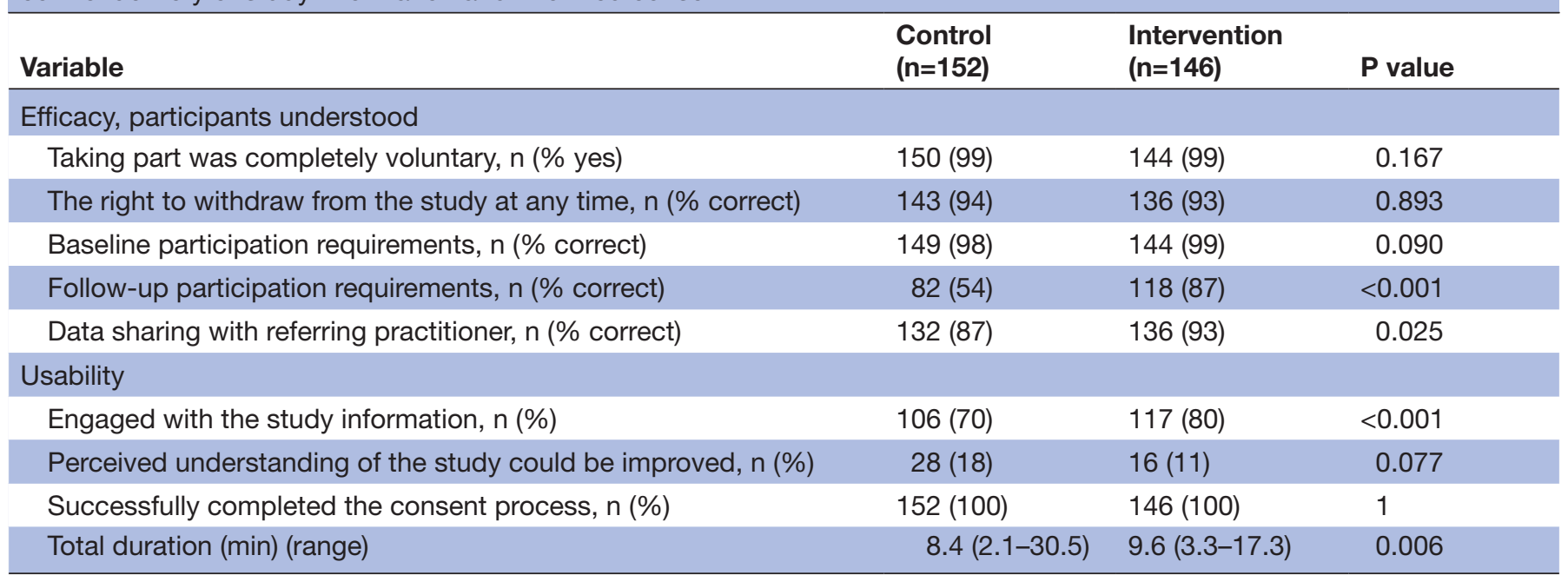

Data are expressed as percentages of the group total. $\mathrm{P}$ values relate to the $\chi^{2}$ test used for the comparison of categorical variables and t-test was used for continuous variables. 
Table 3 Acceptability of participant information and informed consent process of study participants randomised to multimedia intervention or control delivery of participant information and informed consent

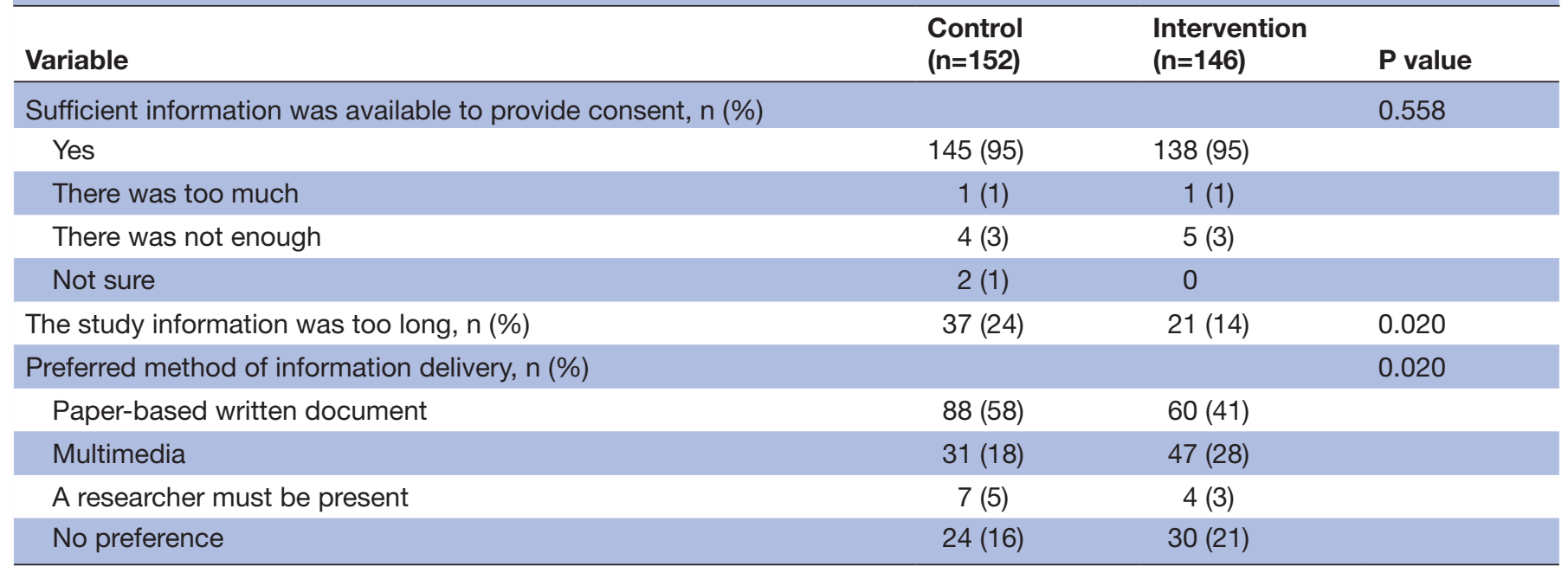

Data are expressed as percentages of the group total. The $\chi^{2}$ test was used for the comparison of categorical variables.

Thirty-seven participants (15 intervention and 22 control) commented on ways to improve their understanding of the study. The themes of these comments focused on simplifying the study information sheet, adding more information to the study postcard, providing a variety of information delivery options for participants to choose from and providing participants with updates on the research outcomes of the study. Four participants in the control group requested assistance with the consent process as they did not have their reading glasses to read the information sheet. No participants in the intervention group requested assistance.

\section{Acceptability of multimedia intervention versus control}

Both groups reported similar levels of acceptability (table 3), although more control participants reported the study information was too long and had a greater preference for paper-based information delivery $(\mathrm{p}=0.020$ for both). Only $4 \%$ of participants reported that a researcher must be present for the consent process and there was no difference between groups.

\section{DISCUSSION}

The key finding from this study was that a self-directed multimedia consent process free from research staff was a suitable mode for delivering study information and obtaining informed consent for a clinical research study. Additionally, multimedia delivery of study information improved participant understanding of aspects of study involvement. High acceptability of both consent processes was reported in this population of middle-toolder aged, community-dwelling adults. These findings suggest that multimedia is an acceptable, efficient and effective alternative to traditional consent processes in medical research.
The evidence on using multimedia to enhance the traditional consent process has concentrated on participants with additional support needs such as low literacy, mental health issues or children. ${ }^{21-24}$ Moreover, previous work focused on augmenting the traditional consent approach with multimedia tools, rather than comparing a truly selfdirected, multimedia consent process, as we have done in this study. Our study design fulfils an identified research gap on the need for high-quality comparisons of selfdirected multimedia delivery of consent compared with the traditional approach for research. ${ }^{17}$ One small study assessed the effectiveness of self-directed multimedia information delivery, but this was in the setting of consent for surgery rather than research participation. In that study, they found that $98 \%$ of multimedia participants understood the information provided compared with $88 \%$ that received conventional verbal consent. ${ }^{18}$ Our findings, in a middle-to-older population without specific support needs, further develop this knowledge beyond a special population and in a larger sample to confirm that a self-directed multimedia platform may be useful among populations without special needs, such as communitydwelling, older adults (ie, average age 63 years). With the potential to enhance current consent processes, further work is needed in diverse populations to investigate the generalisability of multimedia consent processes.

Ethical conduct is paramount in medical research, and consent processes need to adapt to adequately reflect modern attitudes and contemporary research practices. ${ }^{13-15}$ This study is relevant to the calls to update consent guidelines to better support participant autonomy and move away from an unwieldy approach of full disclosure, to one that supports values-based decision making for participants. ${ }^{13526}$ As few as $4 \%$ of participants reported research staff must be present during the consent process. Importantly, we observed starkly different levels 
of participant engagement with study information, with only $9 \%$ of participants in the multimedia group choosing to listen to the separate audio segments on the more technical aspects of research governance. This indicates that engaging with all study information, by reading, watching or listening, is not necessarily a priority for making an autonomous choice for most participants and is highly individual. Accordingly, consent processes, such as we have provided, using self-directed multimedia, should support participant autonomy by providing options to engage with study information relevant to their values to aid decision-making processes.

\section{Implications for research and practice}

A key benefit of the self-directed consent process evaluated in this research is its potential to improve participant understanding of study information while reducing the burden of consent for research staff. Another key advantage is the possible economic benefit. Current healthcare consumers and research participants are highly 'information-savvy' and may seek the delivery of information from different platforms or prefer diverse options for information delivery such as multimedia. ${ }^{27}$ We suggest that the benefits of better delivery of consent information will drive cost savings both in the short and longer terms. Short-term savings include the cost of time and the uptake of information that is more beneficial (and better understood) by the participant including understanding participation requirements. Longer-term savings could include cost savings through widespread uptake of selfdirected multimedia consent processes to reduce staff burden (noting that only four participants asked for staff assistance in our study).

Although attempts at standardisation of conventional paper-based consent processes have been made, achieving standardised consent delivery by study personnel is challenging. ${ }^{128}$ Multimedia tools offer an inherently standardised method of information delivery, as the delivery is predetermined, that would otherwise be difficult to achieve in standard consent processes undertaken in multisite research projects with large staff teams. As demonstrated in this study, a self-directed multimedia consent process allows flexibility to engage with study information relevant to support participant decision making while also ensuring that the delivery of that information is standardised for each participant. Several publicly available software packages support the development and/or delivery of self-directed multimedia consent processes and many can also be used to collect data as we did in this study (eg, Research Electronic Data Capture ${ }^{29}$ posing an attractive alternative to current consent processes. The findings of this present study highlight that self-directed multimedia information delivery achieves desired levels of participant understanding and is as appropriate as the traditional paper-based approach for obtaining participant consent. Indeed, in a number of settings, it may be more desirable, such as large-scale multisite clinical trials. ${ }^{30} 31$

\section{Strengths and limitations}

A key strength of this work is the randomised evaluation design among a sizeable study sample, conducted in a real clinical setting, and demonstrates the value of this approach in a minimal or low-risk research protocol. Further work is needed to explore the acceptability and appropriateness of consent processes independent of research staff before it is implemented for more complex research with higher levels of participant risk. Potential limitations include the possibility of selection bias as participation was by self-selection after initial eligibility screening. We cannot be sure whether the findings will be generalisable beyond our study population of middle-toolder aged, mostly white adults with high levels of educational attainment, and this will need to be tested in the future. Additionally, it was not possible to use validated evaluation tools to assess the efficacy, usability and acceptability of the consent process due to time constraints of undertaking a research protocol within a pathology services setting. It was not feasible to notify participants about the research prior to presenting at pathology services and all participants had to take part on the same day their blood sample was collected. For this reason, the entire process had to be shorter than 20 min to minimise disruption to participants and pathology services. Efficacy, usability and acceptability were assessed of the consent process as a whole and not specifically of the information provided on the second page of the information sheet or the separate audio in the multimedia consent process. Consequently, we cannot draw definitive conclusions on these different aspects of the consent process. Additionally, the duration of video and audio content was not visible to participants before selection, which may have deterred some participants from engaging with this information and should be rectified in the future.

\section{CONCLUSION}

A self-directed, multimedia consent process free from research staff was effective and acceptable to deliver participant information and receive informed consent in a middle-to-older age population. Our findings suggest that multimedia consent processes may be suitable for reducing the burden on research staff and improving the delivery of consent for research.

\section{Twitter Niamh Chapman @nchapmanTAS}

Acknowledgements The authors would like to thank the Diagnostic Service Pty Ltd staff for their expertise and assistance throughout all aspects of this study, with specific thanks to Carol Batt for recruiting participants. They also thank David J Lipscombe, Michelle L Davis Patman and Belinda Kendall-White for providing their time as community research advisors and to graphic designer Rory Dick for his patience and creativity.

Contributors NC, RM, MKA, MGS, MRN and JES made substantial contributions to the conception of the study. NC and MKA analysed the data for the study. NC and RF contributed to the acquisition of data. NC, RM, MKA, MGS, RF, MRN, JAC and JES interpreted the data, drafted the article and revised it critically for intellectual content. All authors contributed to drafts of the article and provided final approval of the version to be published and accept accountability for article integrity. 
Funding This work was supported by a grant from the Royal Hobart Hospital Research Foundation [reference 16-006].

Competing interests None declared.

Patient and public involvement Patients and/or the public were involved in the design, or conduct, or reporting, or dissemination plans of this research. Refer to the Methods section for further details.

\section{Patient consent for publication Not required.}

Ethics approval The study was performed in concordance with ethical approval obtained from Tasmanian Human Research Ethics Committee [H0015648].

Provenance and peer review Not commissioned; externally peer reviewed.

Data availability statement Data are available upon reasonable request. Data are available upon reasonable request to the corresponding author.

Open access This is an open access article distributed in accordance with the Creative Commons Attribution Non Commercial (CC BY-NC 4.0) license, which permits others to distribute, remix, adapt, build upon this work non-commercially, and license their derivative works on different terms, provided the original work is properly cited, appropriate credit is given, any changes made indicated, and the use is non-commercial. See: http://creativecommons.org/licenses/by-nc/4.0/.

\section{ORCID iDs}

Niamh Chapman http://orcid.org/0000-0001-6317-5594

Julie A Campbell http://orcid.org/0000-0002-1820-6758

\section{REFERENCES}

1 NHMRC. National statement on ethical conduct in human research (updated 2018), 2007. Available: https://www.nhmrc.gov.au/aboutus/publications/national-statement-ethical-conduct-human-research2007-updated-2018\#block-views-block-file-attachments-contentblock-1 [Accessed 12 Apr 2019].

2 Department of Health, Education, and Welfare, National Commission for the Protection of Human Subjects of Biomedical and Behavioral Research. The Belmont report. ethical principles and guidelines for the protection of human subjects of research. J Am Coll Dent 2014;81:4-13.

3 WMA - The World Medical Association-WMA Declaration of Helsinki - Ethical Principles for Medical Research Involving Human Subjects. Available: https://www.wma.net/policies-post/wma-declaration-ofhelsinki-ethical-principles-for-medical-research-involving-humansubjects/ [Accessed 3 Apr 2019].

4 Flory J, Emanuel E. Interventions to improve research participants' understanding in informed consent for research: a systematic review. JAMA 2004;292:1593-601.

5 Joffe S, Cook EF, Cleary PD, et al. Quality of informed consent in cancer clinical trials: a cross-sectional survey. Lancet 2001;358:1772-7.

6 Schaeffer $\mathrm{MH}$, Krantz DS, Wichman A, et al. The impact of disease severity on the informed consent process in clinical research. $A m \mathrm{~J}$ Med 1996;100:261-8.

7 Boyd K. The impossibility of informed consent? J Med Ethics 2015;41:44-7.

8 O'Neill O, O'Neill O. Some limits of informed consent. J Med Ethics 2003;29:4-7.

9 Fortun P, West J, Chalkley L, et al. Recall of informed consent information by healthy volunteers in clinical trials. QJM 2008;101:625-9.

10 Crepeau AE, McKinney BI, Fox-Ryvicker M, et al. Prospective evaluation of patient comprehension of informed consent. J Bone Joint Surg Am 2011;93:e114(1)-e114(7).
11 Doshi $\mathrm{P}$, Hur $\mathrm{P}$, Jones $\mathrm{M}$, et al. Informed consent to study purpose in randomized clinical trials of antibiotics, 1991 through 2011. JAMA Intern Med 2017;177:1452-9.

12 US Department of Health and Human Services. Human subjects research protections: enhancing protections for research subjects and reducing burden, delay, and ambiguity for Investigators. Federal Register 2011;76:44512-31.

13 Emanuel EJ, Menikoff J. Reforming the regulations governing research with human subjects. N Engl J Med 2011;365:1145-50.

14 Gainotti S, Turner C, Woods S, et al. Improving the informed consent process in international collaborative rare disease research: effective consent for effective research. Eur J Hum Genet 2016;24:1248-54.

15 Annas GJ. Globalized clinical trials and informed consent. N Engl J Med 2009;360:2050-3.

16 Nishimura A, Carey J, Erwin PJ, et al. Improving understanding in the research informed consent process: a systematic review of 54 interventions tested in randomized control trials. BMC Med Ethics 2013;14:28.

17 Ryan RE, Prictor MJ, McLaughlin KJ, et al. Audio-Visual presentation of information for informed consent for participation in clinical trials. Cochrane Database Syst Rev 2008:CD003717.

18 Cornoiu A, Beischer AD, Donnan L, et al. Multimedia patient education to assist the informed consent process for knee arthroscopy. ANZ J Surg 2011;81:176-80.

19 Chapman N, Fonseca R, Murfett L, et al. Integration of absolute cardiovascular disease risk assessment into routine blood cholesterol testing at pathology services. Fam Pract 2020. doi:10.1093/fampra/ cmaa034. [Epub ahead of print: 16 Apr 2020].

20 National Vascular Disease Prevention Alliance. Guidelines for the management of absolute cardiovascular disease risk. Melbourne: National Heart Foundation of Australia, 2009.

21 Yeh DM, Chun S, Terrones L, et al. Using media to improve the informed consent process for youth undergoing pediatric endoscopy and their parents. Endosc Int Open 2017;5:E41-6.

22 Jeste DV, Palmer BW, Golshan S, et al. Multimedia consent for research in people with schizophrenia and normal subjects: a randomized controlled trial. Schizophr Bull 2009;35:719-29.

23 Ownby RL, Acevedo A, Goodman K, et al. Health literacy predicts participant understanding of orally-presented informed consent information. Clin Res Trials 2015;1:15-19.

24 Afolabi MO, Bojang K, D'Alessandro U, et al. Digitised audio questionnaire for assessment of informed consent comprehension in a low-literacy African research population: development and psychometric evaluation. BMJ Open 2014;4:e004817.

25 Kraft SA, Porter KM, Shah SK, et al. Comprehension and choice under the revised common rule: improving informed consent by offering reasons why some enroll in research and others do not. Am J Bioeth 2017;17:53-5.

26 Sugarman J. Examining provisions related to consent in the revised common rule. Am J Bioeth 2017;17:22-6.

27 Shih Y-CT, Tai-Seale M. Physicians' perception of demand-induced supply in the information age: a latent class model analysis. Health Econ 2012;21:252-69.

28 Weinmeyer R. Lack of standardized informed consent practices and medical malpractice. Virtual Mentor 2014;16:120-3.

29 Harris PA, Taylor R, Thielke R, et al. Research electronic data capture (REDCap)--a metadata-driven methodology and workflow process for providing translational research informatics support. J Biomed Inform 2009;42:377-81.

30 Blake K, Holbrook JT, Antal H, et al. Use of mobile devices and the Internet for multimedia informed consent delivery and data entry in a pediatric asthma trial: study design and rationale. Contemp Clin Trials 2015;42:105-18.

31 Afolabi MO, McGrath N, D'Alessandro U, et al. A multimedia consent tool for research participants in the Gambia: a randomized controlled trial. Bull World Health Organ 2015;93:320-8. 